\title{
Using Monoclonal Fab to Facilitate Single Particle CryoEM Studies of Small Proteins
}

\author{
Shenping $\mathrm{Wu}^{1}$, JungMin $\mathrm{Kim}^{2}$, Charles S. Craik ${ }^{2}$ and Yifan Cheng ${ }^{1}$ \\ 1. The W.M. Keck Advanced Microscopy Laboratory, Department of Biochemistry and Biophysics, \\ University of California San Francisco, 600 16th Street, San Francisco, CA 94158, USA. \\ 2. Department of Pharmaceutical Chemistry, University of California San Francisco, 600 16th Street, San \\ Francisco, CA 94158, USA.
}

As a versatile tool in structural biology, single particle electron cryomicroscopy (cryoEM) has achieved great success in recent years, demonstrated by the determination of near-atomic resolution threedimensional (3D) reconstructions of large protein assemblies with high symmetry [1]. The technological developments in all aspects of cryoEM, from hardware of electron microscopy [2] to software of image processing, have enabled single particle cryoEM to be applicable to a broad range of biological molecules [3]. However, it remains to be a major challenge in single particle cryoEM to determine highresolution 3D reconstruction of small macromolecular complexes between 100 to $300 \mathrm{kDa}$ without high symmetry. Small protein complexes are difficult to be visualized by cryoEM. Iterative refinement procedures in determining $3 \mathrm{D}$ reconstruction make single particle cryoEM very sensitive to model induced bias. Thus validation of 3D reconstruction of small protein complexes is challenging, particularly for small proteins without distinct structural features that can be used to validate the 3D reconstruction.

We have developed a novel technique to facilitate determination of reliable 3D reconstructions of small protein complexes by single particle cryoEM [4]. The overall strategy is to use one or more monoclonal Fabs to form a stable and rigid complex with the target protein to facilitate structural analysis of the small target protein complexes by single particle cryoEM. The rationale of this approach is as follows: Formation of a Fab-protein complex increases the effective size of the target protein and the increased size and unique shape of the Fab makes it easier to be visualized by cryoEM. All Fabs have characteristic shapes with a variable domain linked to a constant domain by a short linker. Therefore, a $\mathrm{Fab}$ bound to a target protein can be easily recognized in raw images as well as in two-dimensional (2D) class averages, with two typical views: one resembles a triangle with a hole in the middle, and the other resembles a dumbbell, defined by two lobes (Figure 1). Such a recognizable feature added to a small target protein provides a fiducial marker for more accurate image alignment of otherwise featureless small proteins. Furthermore, the presence of a distinct feature of a Fab in the 3D reconstruction provides strong evidence for the correctness of the structure and serves as a validation criterion.

We show in Figure 1 an example to demonstrate our approach. Human proprotein convertase subtilisin/kexin type 9 (PCSK9) is a key regulator of serum hepatocyte low-density lipoprotein receptor (LDL-R) expression. This small protein has a molecular weight of only $\sim 70 \mathrm{kDa}$, and its $2 \mathrm{D}$ class averages calculated from negatively stained particle do not contain sufficient structural features to enable identification of its individual sub-domains (Figure 1a-c). On the contrary, negative stain EM of a Fab always shows distinct shapes (Figure 1d-f). As a complex, the Fab-PCSK9 has a total molecular weight of $\sim 130 \mathrm{kDa}$, and its negative stain $2 \mathrm{D}$ class averages show clear structural features that can be recognized easily. We have used this approach to determine 3D reconstructions of a number of small proteins complexes, including Fab-PCSK9, Fab - HIV integrase complexes. 
References:

[1] X Zhang et al, Cell 141 (2010) p472.

[2] X Li et al, Nature Methods (2013) Submitted.

[3] SH Scheres and S Chen, Nature Method 9 (2012) p. 854.

[4] S Wu et al, Structure 20 (2012) p. 582.

[5] The authors acknowledge funding from the National Institute of Health, R01GM098672 (YC), P50GM082250 (YC and CSC) and P50 GM073210 (CSC).
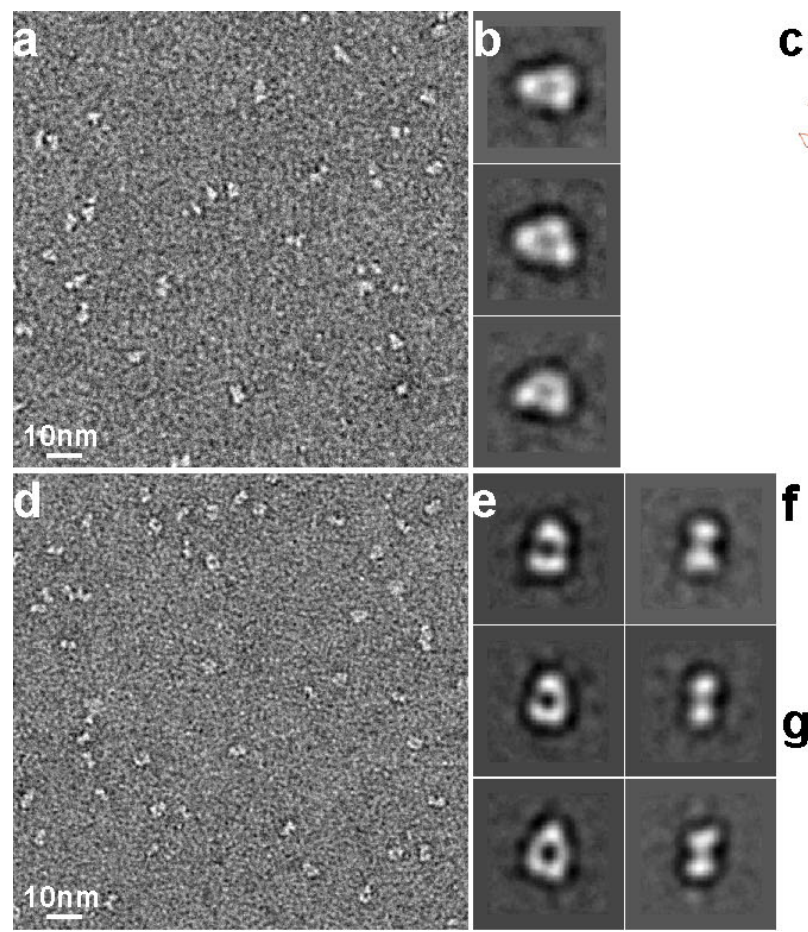
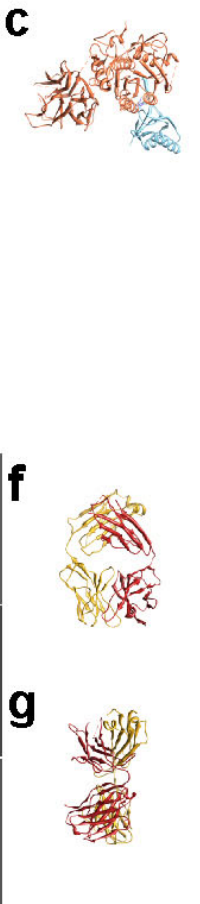
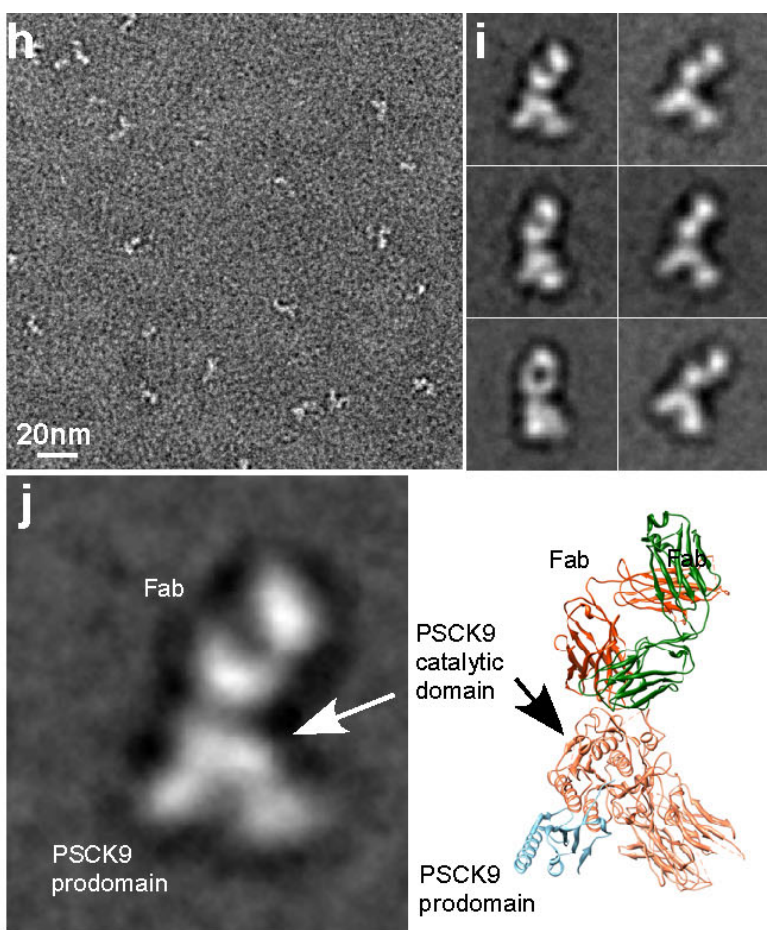

Figure 1. Negative stain EM study of PCSK9, Fab J16 and their complex. (a, b, c) An EM image of negatively stained PCSK9, its representative class averages and ribbon diagram of human PCSK9 (pdb code: 2P4E). (d, e, f, g) An EM image of negatively stained Fab J16, its representative class averages and two different views of a typical Fab (pdb code: 1M71) in a ribbon diagram corresponding to the class averages shown in e. (h, i) An EM image of PCSK9-Fab complex and its typical and representative class averages. (j) On the left is an enlarged view of a 2D class average of PCSK9-Fab complex, same as the top left class average shown in panel i. On the right is a ribbon diagram in which a PCSK9 and a Fab were placed together with their orientations closely matching that of the class average on the left. Domains of PCSK9 and Fab are indicated. 